\title{
Interlinked temporal changes in environmental conditions, chemical characteristics of sediments and macrofaunal assemblages in an estuarine intertidal sandflat (Seto Inland Sea, Japan)
}

Paolo Magni • Serena Como • Shigeru Montani • Hiroaki Tsutsumi

Published online: 19 July 2006

(C) Springer-Verlag 2006

Marine Biology DOI 10.1007/s00227-006-0298-0

Unfortunately Table 5 contained errors. The correct Table 5 is shown below.

The online version of the original article can be found at http://dx.doi.org/10.1007/s00227-006-0298-0.

P. Magni $(\varangle) \cdot$ S. Como

IMC - International Marine Centre, Località Sa Mardini,

Torregrande, 09072 Oristano, Italy

e-mail: p.magni@imc-it.org

S. Montani

Graduate School of Environmental Science,

Hokkaido University, Kita 13 Nishi 8,

Kita-ku, Sapporo,

060-0813 Hokkaido, Japan

H. Tsutsumi

Faculty of Environmental \& Symbiotic Sciences,

Prefectural University of Kumamoto, 3-1-100 Tsukide,

863-8502 Kumamoto, Japan 
Table 5 (a) Analyses of variance for the abundance of Cirriphormia tentaculata for differences among dates, and SNK test. (b) Summary of analyses of variance and SNK test for the taxa contributing most for the differences among dates in terms of abundances and biomass (SIMPER, cut-off 70\%)

\begin{tabular}{|c|c|c|c|c|c|}
\hline Source & $d f$ & MS & $F$ & $P$ & SNK test \\
\hline \multicolumn{6}{|l|}{ (a) C. tentaculata } \\
\hline Date & 4 & 4.14 & 5.58 & 0.0006 & $\mathrm{Su}<\mathrm{Sp}_{94}=\mathrm{A}=\mathrm{W}=\mathrm{Sp}_{95}$ \\
\hline Residual & 70 & 0.74 & & & \\
\hline \multirow[t]{2}{*}{ Total } & 74 & & & & \\
\hline & & MS & $F_{4,70}$ & $P$ & SNK test \\
\hline \multicolumn{6}{|l|}{ (b) } \\
\hline \multicolumn{6}{|l|}{ Abundances } \\
\hline R. philippinarum & & 9.80 & 13.41 & 0.0000 & $\mathrm{~A}<\mathrm{W}=\mathrm{Sp}_{94}=\mathrm{Su}=\mathrm{Sp}_{95}$ \\
\hline Gammaridae sp. & & 6.20 & 19.36 & 0.0000 & $\mathrm{Sp}_{94}=\mathrm{A}<\mathrm{W}=\mathrm{Su}=\mathrm{Sp}_{95}$ \\
\hline Polydora sp. & & 9.57 & 8.25 & 0.0000 & NAH \\
\hline Spionidae sp. & & 17.74 & 6.17 & 0.0003 & $\mathrm{Su}>\mathrm{Sp}_{94}=\mathrm{A}=\mathrm{W}=\mathrm{Sp}_{95}$ \\
\hline Dimorphostylis sp. & & 17.39 & 10.38 & 0.0000 & $\mathrm{Sp}_{94}>\mathrm{Su}>\mathrm{A}=\mathrm{W}=\mathrm{Sp}_{95}$ \\
\hline Capitella sp. & & 69.84 & 12.98 & 0.0000 & $\mathrm{Su}=\mathrm{Sp}_{94}=\mathrm{A}=\mathrm{W}<\mathrm{Sp}_{95}$ \\
\hline M. senhousia & & 1.51 & 0.96 & 0.4348 & NS \\
\hline C. erithraeensis & & 1.32 & 0.97 & 0.4294 & NS \\
\hline A. oxycephala & & 0.54 & 1.05 & 0.3893 & NS \\
\hline C. muromiensis & & 0.43 & 2.35 & 0.0627 & NS \\
\hline Nephtys sp. & & 0.25 & 1.71 & 0.1583 & NS \\
\hline \multicolumn{6}{|l|}{ Biomass } \\
\hline M. senhousia & & 1.50 & 3.99 & 0.0056 & $\mathrm{NAH}$ \\
\hline R. philippinarum & & 1.15 & 7.88 & 0.0000 & $\mathrm{Su}>\mathrm{Sp}_{94}=\mathrm{A}=\mathrm{W}=\mathrm{Sp}_{95}$ \\
\hline C. tentaculata & & 0.18 & 7.31 & 0.0001 & $\mathrm{Su}=\mathrm{A}<\mathrm{Sp}_{94}=\mathrm{W}=\mathrm{Sp}_{95}$ \\
\hline C. erithraeensis & & 0.11 & 1.76 & 0.1464 & NS \\
\hline Polydora sp. & & 0.07 & 5.68 & 0.0005 & $\mathrm{Su}=\mathrm{Sp}_{94}=\mathrm{A}=\mathrm{W}<\mathrm{Sp}_{95}$ \\
\hline
\end{tabular}

$N A H$ no alternative hypothesis, $N S$ no significant effects 